\title{
Phosphorylation in vivo of the $\mathrm{P}$ light chain of myosin in rabbit fast and slow skeletal muscles
}

\author{
Sara A. WESTWOOD,* Olga HUDLICKA $\dagger$ and S. Victor PERRY* \\ Departments of ${ }^{*}$ Biochemistry and $\dagger$ Physiology, University of Birmingham, P.O. Box 363, \\ Birmingham B15 2TT, U.K.
}

(Received 16 September 1983/Accepted 17 November 1983)

1. The $\mathbf{P}$ light chain of myosin is partially phosphorylated in resting slow and fast twitch skeletal muscles of the rabbit in vivo. The extent of $\mathrm{P}$ light-chain phosphorylation increases in both muscles on stimulation. 2. Rabbit slow-twitch muscles contain two forms of the P light chain that migrate with the same electrophoretic mobilities as the two forms of $P$ light chain in rabbit ventricular muscle. 3. The rate of phosphorylation of the $\mathrm{P}$ light chain in slow-twitch muscle is slower than its rate of phosphorylation in fast-twitch muscles during tetanus. 4. The rate of $P$ light-chain dephosphorylation is slow after tetanic contraction of fast-twitch muscles in vivo. The time course of dephosphorylation does nat correlate with the decline of post-tetanic potentiation of peak twitch tension in rabbit fast-twitch muscles. 5. The frequency of stimulation is an important factor in determining the extent of $\mathrm{P}$ light-chain phosphorylation in fast- and slow-twitch muscles.

Phosphorylation of the $\mathrm{P}$ \{regulatory or DTNB [5,5'-dithiobis-(2-nitrobenzoic acid)] $\}$ light chain of myosin from skeletal muscle occurs by the action of the highly specific enzyme myosin light-chain kinase (Pires \& Perry, 1977). The enzyme requires calmodulin as an essential cofactor and is activated at $\mathrm{Ca}^{2+}$ concentrations similar to those that initiate contraction (Yagi et al., 1978; Nairn \& Perry, 1979). Although stimulation leads to light-chain phosphorylation, it is very unlikely in striated muscle that a phosphorylation-dephosphorylation cycle could occur synchronously with the crossbridge cycle (Perry et al., 1978). In fast skeletal muscle, the striated muscle in the rabbit with the highest myosin light-chain kinase activity, the activity per unit weight of tissue is only 1 or $2 \%$ of that of the $\mathrm{Mg}^{2+}$-stimulated myofibrillar ATPase, and the light-chain phosphatase is much less active than the kinase. These facts, coupled with the highly specific nature of the two enzymes and their widespread distribution, imply that the role of phosphorylation of the $\mathbf{P}$ light chain in striated muscle is to modulate the contractile response rather than constituting an essential step in excitation-contraction coupling.

In resting skeletal muscle the $P$ light chain is partially phosphorylated, although values reported in

Abbreviations used: TA, tibialis anterior; EDL, extensor digitorum longus. the literature range from 10 to $50 \%$ (Barany \& Barany, 1977; Stull \& High, 1977; Barany et al., 1979; Manning \& Stull, 1979; Barany et al., 1982; Kushmerick \& Crow, 1982). There are several reports (Stull \& High, 1977; Manning \& Stull, 1979) that stimulation of fast skeletal muscle increases the phosphorylation up to close to $100 \%$ if prolonged, although Barany et al. (1982) found in chicken muscle that phosphorylation did not exceed $50 \%$ even after prolonged isometric contraction. The variation between the values for Plight-chain phosphorylation in apparently similar experimental conditions reported by different investigators may reflect muscle and species differences and whether the muscle is stimulated in situ or in vitro.

Manning \& Stull (1979) consider that light-chain phosphorylation plays a role in post-tetanic potentiation in fast skeletal rat muscle, whereas Kushmerick \& Crow (1982) suggest that it is responsible for the decreased velocity of shortening that occurs after continued activity in mouse fasttwitch muscle. On the other hand, Butler et al. (1983) do not report a correlation between P-lightchain phosphorylation and the velocity of shortening in this muscle.

Both of these physiological properties would be expected to be reflected in the effect of phosphorylation on the enzymic behaviour of $\mathrm{Mg}^{2+}$-dependent ATPase of isolated actomysin. Unfortu- 
nately, here also the results are somewhat equivocal. At low ionic strength, slight or no activation of the ATPase on phosphorylation of the myosin has been reported (Morgan et al., 1976; Cooke et al., 1981), but Pemrick (1980) obtained much higher activation under certain conditions. On the other hand, it has been reported that, if myofibrils are lightly cross-linked with glutaraldehyde, phosphorylation of the $\mathrm{P}$ light chain leads to a fall in $V_{\max .}($ Cooke et al., 1982). This would correlate well with the fall in maximum velocity of shortening observed after tetanus in mouse fast-twitch muscle (Kushmerick \& Crow, 1982).

These somewhat inconsistent findings may be a consequence of the differences in the prior treatment of the muscle and accurately measuring the extent of P-light-chain phosphorylation. The effect of stimulation in situ on the phosphorylation of the $P$ light chain of fast and slow skeletal muscles of the rabbit in vivo has therefore been undertaken by using the high resolution of two-dimensional electrophoresis and procedures that avoid breakdown of $P$ light chain during analysis. Some of the findings have been briefly reported elsewhere (Westwood et al., 1982).

\section{Methods}

\section{Preparation of muscles and stimulation}

Experiments were performed on New Zealand White rabbits of either sex $(2.5-3.5 \mathrm{~kg}$ body wt.) under fluothane anaesthesia. Drills inserted into the lower (distal) part of the femur and tibia ensured good fixation for recording of isometric tension of either fast (TA and EDL), or slow (soleus) muscles whose tendons were cut and connected to an Ether strain-gauge Dynamometer type UF2 (Pye Ether, Stevenage, Herts., U.K.). The tension was recorded on a Devices recorder. In all cases at least $45 \mathrm{~min}$ elapsed after anaesthesia before stimulation or resting extents of phosphorylation were determined. All muscles were stimulated via their nerves, and the optimal length was set with about five consecutive twitches. The period between the adjustment and the beginning of stimulation was about $10 \mathrm{~min}$. Muscles were stimulated for different periods of time to produce either unfused tetanic contractions $(60 \mathrm{~Hz}$ for fast, $30 \mathrm{~Hz}$ for slow muscles) or at different frequencies to produce single twitches, by using $0.1 \mathrm{~ms}$ pulse width and supramaximal voltage (usually $3-5 \mathrm{~V}$ ), with a Grass S8 stimulator.

Preparation of muscle extracts for determination of $P$ light-chain phosphorylation

Muscles were frozen in situ at the times and under the conditions described in the text by the application of large Wollenberg clamps previously stored in liquid $\mathbf{N}_{2}$. The tendons of the muscles frozen in the clamps were cut and the muscles immediately transferred to liquid $\mathrm{N}_{2}$. Within $2 \mathrm{~h}$ approx. 3-5g of frozen muscle was rapidly homogenized in $20 \mathrm{ml}$ of ice-cold $15 \%(\mathrm{w} / \mathrm{v})$ trichloroacetic acid at $0^{\circ} \mathrm{C}$ in a small high-speed Waring blender-type homogenizer. The precipitation with trichloroacetic acid was essential to destroy endogenous protein phosphatase activity (Westwood \& Perry, 1981). The precipitated protein was dissolved in urea, and the solution was neutralized and prepared for two-dimensional electrophoresis by the procedure of Anderson \& Anderson $(1978 a, b)$ as described by Westwood \& Perry (1981).

The extent of phosphorylation of the $P$ light chain was determined from two-dimensional gels be scanning with a densitometer and cutting out and weighing the traces (Westwood \& Perry, 1981).

\section{Results}

Two-dimensional electrophoresis of skeletal-muscle extracts

When extracts prepared from the samples of resting skeletal muscle were examined by twodimensional electrophoresis in a restricted gradient of $\mathrm{pH} 4-6$, a good separation of the phosphorylated and non-phosphorylated forms of the $P$ light chain of myosin was obtained (Fig. 1). The location of the spots corresponding to the $\mathbf{P}$ light chains was established by their mobilities in relation to other spots corresponding to known proteins on the two-dimensional electrophoretogram and by comparison with those observed on electrophoresis of samples of rabbit skeletal myosin that had been phosphorylated in vitro with myosin light-chain kinase (Westwood \& Perry, 1981). In early control experiments, the muscles were removed separately for P-light-chain analysis without the surgery required to mount them for recording and without the initial stimulation required to set the optimal length. The values obtained for the individual EDL and TA muscles were not significantly different from those obtained when the EDL and TA muscles were frozen together. For most of the experiments the muscles were frozen together to speed up the procedure and to avoid any unnecessary manipulation of the two muscles that might lead to stimulation.

Whereas only two spots corresponding to the $\mathbf{P}$ light chain and its phosphorylated form were observed with the EDL/TA muscle extracts, with soleus muscle four spots were invariably present (Fig. 1). In resting soleus muscle the two most basic spots represented the major forms of the $P$ light chain, and the two fainter, more acidic, spots rep- 


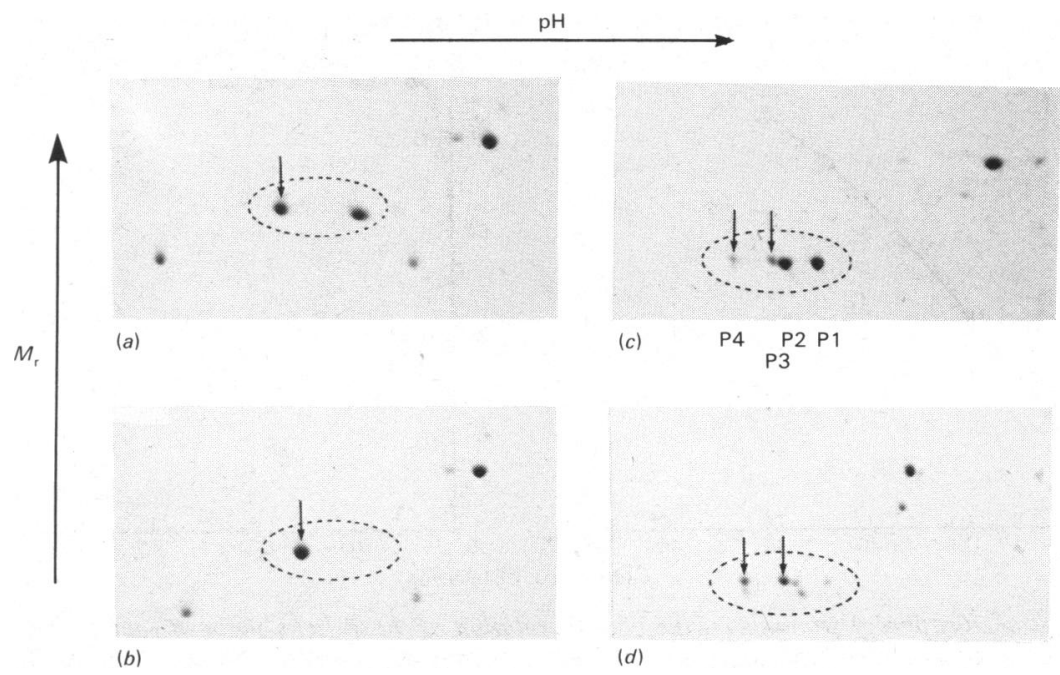

Fig. 1. Two-dimensional electrophoresis of the light chains of myosin in resting and stimulated rabbit skeletal muscles The Figure shows regions of the two-dimensional electrophoretogram, obtained as described in the Materials and methods section, that contain the myosin light chains. They represent regions containing proteins with $M_{\mathrm{r}}$ in the range $15000-30000$ and with $\mathrm{pI}$ values in the range 4.8-5.5. The $P$ light chains are enclosed in dashed ellipsoids, and arrows indicate the phosphorylated forms of the P light chains. P1 and P2 are the two forms of the P light chains in soleus, and P3 and P4 are respectively their phosphorylated derivatives. $(a)$ Resting EDL/TA muscles; $(b)$ EDL/TA muscles after $13 \mathrm{~s}$ at $60 \mathrm{~Hz}$; (c) resting soleus muscle; (d) soleus muscle after $24 \mathrm{~s}$ at $30 \mathrm{~Hz}$.

resented less than $20 \%$ of the total $P$ light-chain fraction. The two more acidic spots were presumed to represent the phosphorylated forms by the similarity of the P-light-chain pattern of soleus muscle to that of rabbit heart, in which tissue the P light chain exists in two isotypic forms. This view was confirmed by the fact that the amounts of the two acidic forms increased at the expense of the basic forms on stimulation (Fig. 1). When rabbit cardiac extracts were added to the soleus-muscle extracts, the cardiac P light chains P1, P2, P3 and P4 (Westwood \& Perry, 1981, 1982) migrated with mobilities identical with those of the four P lightchain spots present in the soleus-muscle extracts. A similar nomenclature to that employed for the heart was therefore adopted for the $P$ light chain in soleus muscle (Fig. 1). In adult rabbit soleus muscle the P2 light chain and its phosphorylated form P4 represented $43 \pm 0.8 \%$ (S.E.M., $n=17$ ) of the total light-chain fraction.

The results in Table 1 indicate that the extent of phosphorylation of the $\mathbf{P}$ light chain of myosin in resting fast-twitch muscles was significantly higher than in the soleus. As the extent of phosphorylation roughly paralleled the myosin light-chain kinase activities of the two types of muscle, it was considered that the difference might be caused in part by the activation of the myosin light-chain kinase owing to a rise in sarcoplasmic $\mathrm{Ca}^{2+}$ concentration as a consequence of the freeze-clamping proce-
Table 1. Extents of phosphorylation of the P light chains of myosin in resting rabbit striated muscle in vivo

Results are percentages, expressed as means \pm S.E.M. for the numbers of different experiments shown in parentheses. Values for soleus and cardiac muscles include values for both forms of the P light chain.

\begin{tabular}{llc}
\multicolumn{1}{c}{ Muscle } & Type & Phosphorylation $(\%$ \\
Skeletal & & \\
Combined EDL/TA & Fast & $50.4 \pm 3.4(11)$ \\
Rectus abdominis & Fast & $54.8 \pm 2.6(4)$ \\
Soleus & Slow & $17.2 \pm 1.0(6)$ \\
Cardiac* & & $23.0 \pm 3.0(4)$
\end{tabular}

* Normal beating heart in the anaesthetized animal (Westwood \& Perry, 1981).

dure. This effect must be small, as clamps chilled in liquid $\mathrm{N}_{2}$ were applied to the intact muscle before removal from the animal and the frozen muscle was immediately immersed and stored in liquid $\mathrm{N}_{2}$ before processing for electrophoresis.

Effect of the duration of tetanus on the phosphorylation of the P light chain in rabbit fast-twitch and slowtwitch skeletal muscles

In combined EDL and TA muscles the maximum extent of phosphorylation $(98 \%)$ was reached after $5 \mathrm{~s}$ tetanus at $60 \mathrm{~Hz}$ (Fig. $2 b$ ). This extent of phosphorylation was maintained if the tetanus was prolonged to $50 \mathrm{~s}$. The maximum extent of P.light-

Vol. 218 


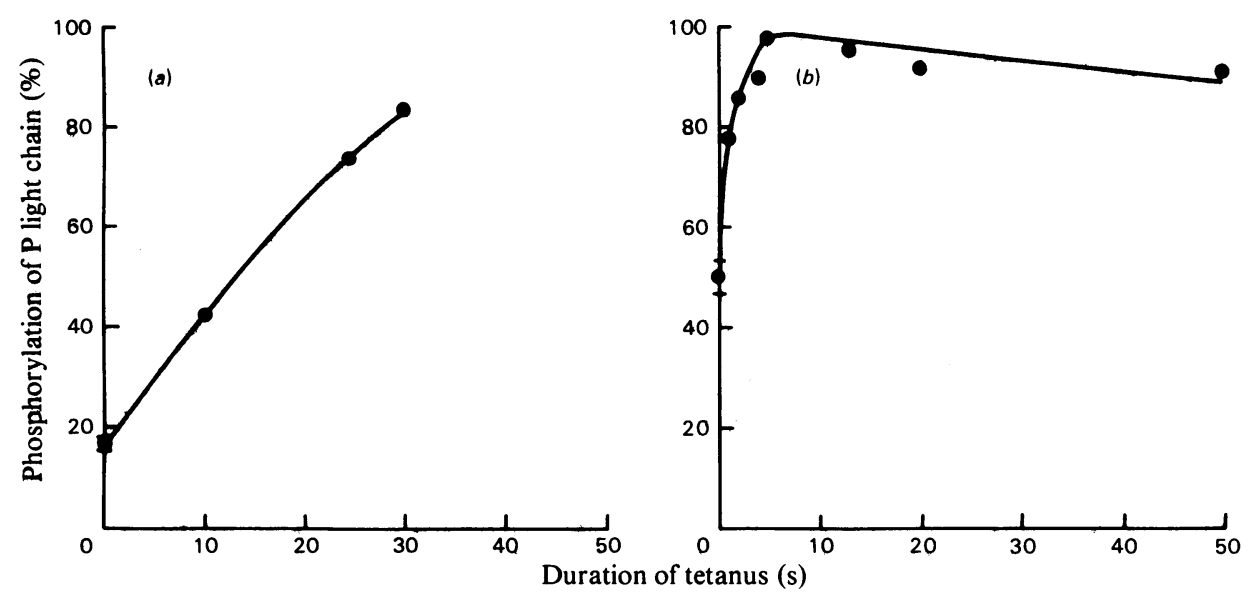

Fig. 2. Effect of duration of stimulus on the phosphorylation of the P light chain of rabbit skeletal muscles Muscles were stimulated isometrically and freeze-clamped at times indicated. (a) Soleus muscle stimulated at $30 \mathrm{~Hz}$; (b) combined EDL/TA muscles stimulated at $60 \mathrm{~Hz}$.

chain phosphorylation in soleus muscle $(80 \%)$ was obtained after $30 \mathrm{~s}$ tetanus at $30 \mathrm{~Hz}$ (Fig. $2 a$ ). Further stimulation at that frequency caused the soleus muscle to become fatigued, and tension fell, as did the extent of P-light-chain phosphorylation.

The rate of P-light-chain phosphorylation in response to stimulation was slower in the soleus than in the combined EDL/TA muscles. A $15 \mathrm{~s}$ tetanus at $30 \mathrm{~Hz}$ of the soleus muscle resulted in $50 \%$ phosphorylation of $\mathbf{P}$ light chains, whereas a similar period of stimulation at $20 \mathrm{~Hz}$, a frequency at which the contractions were not fused, resulted in $90 \%$ phosphorylation in fast muscle.

Post-tetanic potentiation and P-light-chain phosphorylation in rabbit fast skeletal muscle

Repeated stimulation of fast-twitch muscle leads to post-tetanic potentiation, i.e. the increase in tension developed in a single twitch after a short period of tetanus. In the combined rabbit EDL/TA muscle the peak twitch tension increased by over $100 \%$ within $10 \mathrm{~s}$ of the tetanus, but the singletwitch response had returned to the pre-tetanic value within $5 \mathrm{~min}$. Tetanic stimulation for $5 \mathrm{~s}$ at $60 \mathrm{~Hz}$ resulted in approx. $100 \%$ phosphorylation of the $\mathbf{P}$ light chain. After tetanus ceased, dephosphorylation proceeded relatively slowly (Fig. 3). Although there was some correlation between the fall in post-tetanic potentiation and P-light-chain phosphorylation for the first $2 \mathrm{~min}$ after tetanus, this was not the case throughout recovery. In the later stages the extent of phosphorylation fell much more slowly than post-tetanic potentiation and was still $130-140 \%$ of the resting value after $15 \mathrm{~min}$, when the tension had returned to the pre-tetanic value. Extrapolation from the rate of dephos-

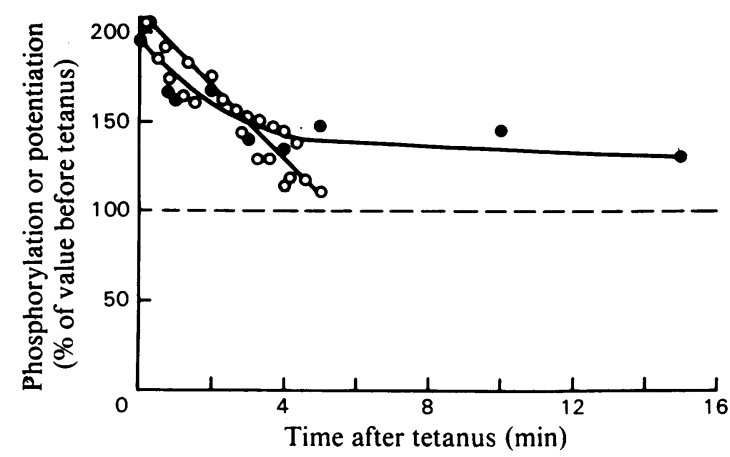

Fig. 3. Phosphorylation of the $P$ light chain and posttetanic potentiation after isometric tetanus of rabbit fast skeletal muscle

Combined EDL/TA muscles were stimulated for $5 \mathrm{~s}$ at $60 \mathrm{~Hz}$. The peak tension of single twitch was measured at different times after tetanus, and muscles were immediately freeze-clamped. Tensions are expressed as percentages of the average value for a single twitch before tetanus, and extents of phosphorylation as a percentage of the resting value (Table 1). , P-light-chain phosphorylation; $\bigcirc$, post-tetanic potentiation.

phorylation occurring 5-15 min after tetanus by using the data shown in Fig. 3 suggests that it would take $45 \mathrm{~min}$ for the $P$ light chain to return to the resting value of phosphorylation.

Effect of frequency of stimulation on P-light-chain phosphorylation of rabbit fast- and slow-twitch muscles

Phosphorylation of the P light chain of EDL/TA muscles was obtained with stimulation at lower 
frequencies producing single twitches. The extent of phosphorylation was dependent not on the total number of impulses received by the motor endplate, but on the frequency at which they were applied (Fig. 4). Electrical stimulation below $1 \mathrm{~Hz}$ failed to produce a marked increase in the extent of $\mathrm{P}$-light-chain phosphorylation in fast muscles even after $30 \mathrm{~min}$ of stimulation, e.g. stimulation at $0.5 \mathrm{~Hz}$ for $30 \mathrm{~min}$, equivalent to 900 impulses, increased the extent of phosphorylation to $10 \%$ above the unstimulated values. In contrast, stimulation at $4 \mathrm{~Hz}$ for $30 \mathrm{~s}$, producing only $120 \mathrm{im}$ pulses, increased the extent of phosphorylation to $80 \%$ in EDL/TA muscles.

It is well known that the repetitive stimulation of fast-twitch muscle leads to a gradual increase in the peak tension of the isometric twitch. Also the time taken to reach peak tension is in inverse relationship to the frequency of stimulation. In view of the results of the effect of frequency of stimulation on P-light-chain phosphorylation described above, a similar relationship should exist between the extent of P-light-chain phosphorylation and the time required to develop peak tension when muscle is stimulated at different frequencies. The results illustrated in Fig. 5 indicate that this is the case. In these experiments, the muscles were freezeclamped at the time at which peak tension was developed in response to electrical stimulation.

If the soleus muscle was stimulated at the natural frequency $(10 \mathrm{~Hz})$ for $30 \mathrm{~s}$, it was $50 \%$ phosphorylated, and little change in phosphorylation occurred

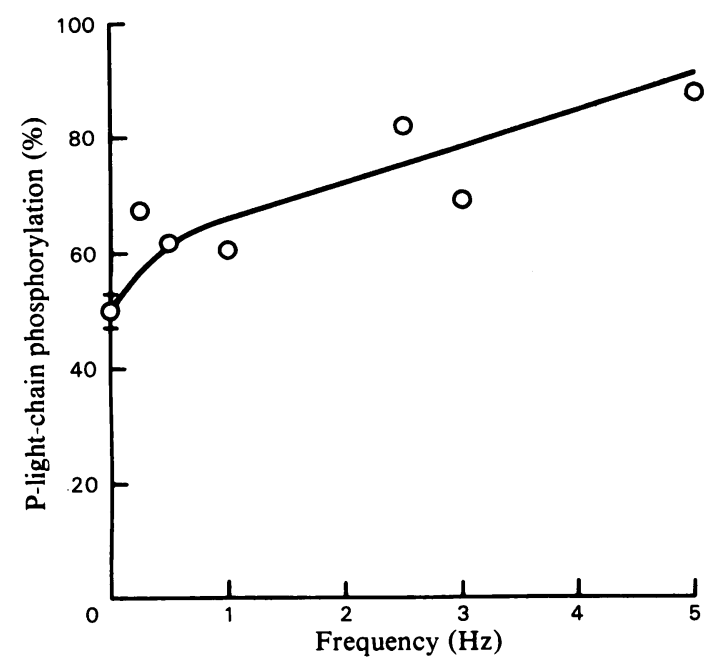

Fig. 4. Effect of frequency of stimulation on the phosphorylation of the $P$ light chain of myosin in rabbit fast skeletal muscles

Combined EDL/TA muscles were stimulated isometrically at frequencies indicated for $60 \mathrm{~s}$ and immediately freeze-clamped. if stimulation was maintained for $4 \mathrm{~min}$. This suggests that the frequency of stimulation rather than the duration of stimulation was important in determining the extent of P-light-chain phosphorylation in slow muscle.

\section{Discussion}

The resting values for the extents of phosphorylation of the P light chain of myosin from rabbit fast skeletal muscle in vivo are high compared with those reported for isolated rat and chicken muscles (Stull et al., 1983; Barany et al., 1982), but similar to that reported for the rabbit by Stull \& High (1977). The different values may indicate species differences, but the possibility exists that some stimulation of the kinase occurs on fixing the muscle for analysis. It is considered that this effect must be minimal in the experiments reported here, for the muscles were rapidly cooled to liquid- $\mathrm{N}_{2}$ temperatures in the present investigation. Also the stimulation experiments indicate that the response of phosphorylation to stimulation is relatively slow. In addition to inactivating the kinase, it is equally important to ensure that the light-chain phosphatase is completely destroyed before extracts are processed for electrophoresis. The phosphatase has been shown to be active in $8 \mathrm{M}$-urea (Westwood \& Perry, 1981), and it is possible that some of the low values reported in the literature are due to dephosphorylation by this enzyme during processing the light chains for analysis.

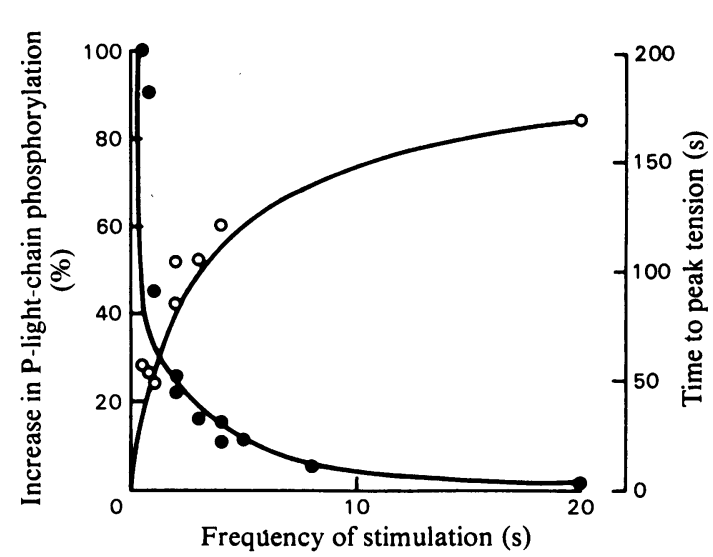

Fig. 5. Effect of frequency of stimulation on the time to reach maximum tension and the extent of phosphorylation of the $P$ light chain

Combined EDL/TA muscles were contracting isometrically at frequencies indicated until maximum tension was developed. Muscles were then immediately freeze-clamped. $\mathrm{O} \%$ increase in P-light-chain phosphorylation; $\mathrm{O}$, time to reach maximum twitch tension. 
In contrast with the report of Barany et al. (1982) that phosphorylation does not exceed $50 \%$ in isolated chicken skeletal muscles, despite repeated stimulation, phosphorylation approaching $100 \%$ was obtained with rabbit fast muscles. In view of the fact that in studies of isolated myosin and actomyosin systems $100 \%$ phosphorylation of the $P$ light chain is readily obtained, it seems surprising that, provided that adequate stimulation is given, $100 \%$ phosphorylation of the light chains should not occur in vivo. Indeed our results and those of Stull \& High (1977) indicate that, in the rabbit at least, this is the case.

In view of the failure of some workers (Kushmerick \& Crow, 1982) to report the phosphorylation after stimulation of the P light chains of slow mammalian skeletal muscle, but not in the chicken (Barany et al., 1982), the results with rabbit soleus are of interest. The polymorphism of the $\mathbf{P}$ light chain appears to be a characteristic of slow skeletal muscle, for no evidence was obtained for the existence of isotypes of this light chain in the EDL or TA muscles. In this respect the soleus muscle resembles the heart, and it may be significant that polymorphism of the $P$ light chain is a feature of the slower types of striated muscle. The relatively high proportion of the total light-chain fraction represented by the $\mathbf{P} 2$ form indicates that it is not solely located in the different cell types, i.e. true type II representing 4.5\% (Gray \& Renkin, 1978), or 'intermediate' type, representing $18.5 \%$ of the total (Dhoot et al., 1981), that have been identified in the adult rabbit soleus muscle. It seems likely that both $\mathrm{P} 1$ and $\mathrm{P} 2$ light chains are present in all type-I cells of the soleus.

It appears that in both fast and slow muscles a certain minimum frequency of stimulation is required to produce significant phosphorylation of the $\mathbf{P}$ light chain. This is presumably related to the frequency of stimulation required to maintain the steady-state $\mathrm{Ca}^{2+}$ concentration of the sarcoplasm at a high enough value to activate the myosin lightchain kinase so that the rate of phosphorylation is much greater than the rate of dephosphorylation catalysed by the myosin light-chain phosphatase. This follows from the close relationship that exists between the extent of phosphorylation and time taken to reach peak twitch tension in fast muscle and frequency of stimulation (Fig. 5). Similar observations have been reported by Stull et al. (1983).

The role of P-light-chain phosphorylation in skeletal muscle still remains uncertain. Although the correlation between phosphorylation and posttetanic potentiation was quite good at higher degrees of phosphorylation, the potentiation had disappeared within $6 \mathrm{~min}$, whereas the phosphorylation was still $40 \%$ above the resting value $16 \mathrm{~min}$ after the tetanus. Stull et al. (1983) have described a similar discrepancy in isolated rat EDL with potentiation ending at $100 \mathrm{~s}$, at which time there was $50 \% \mathrm{P}$-light-chain phosphorylation. The faster return of tension to pre-tetanic values in their experiments is probably due to the fact that they worked with isolated muscle at $23^{\circ} \mathrm{C}$.

The observation that light-chain phosphorylation occurred in soleus muscle, which does not exhibit post-tetanic potentiation (Close \& Hoh, 1969), makes it very unlikely that the latter effect is a direct consequence of phosphorylation of myosin, as has been implied by Manning \& Stull (1979). Indeed, the observation that P-light-chain phosphorylation occurred in both fast and slow muscle types would suggest that any physiological property that is unique to either fast or slow muscle is not a consequence of P-light-chain phosphorylation.

This work was supported in part by a grant from the Medical Research Council.

\section{References}

Anderson, N. G. \& Anderson, N. L. (1978a) Anal. Biochem. 85, 331-340

Anderson, N. L. \& Anderson, N. G. (1978b) Anal. Biochem. 85, 341-354

Barany, K. \& Barany, M. (1977) J. Biol. Chem. 252, 4751-4754

Barany, K., Barany, M., Gillis, J. M. \& Kushmerick, M. J. (1979) J. Biol. Chem. 254, 3617-3623

Barany, K., Vandermeulen, D. L., Ledvora, F. G. \& Barany, M. (1982) Arch. Biochem. Biophys. 217, 392396

Butler, T. M., Siegman, M. J., Mooers, S. V., Barsott, R. J. (1983) Science 220, 1167-1169

Close, R. \& Hoh, J. F. Y. (1969) Nature (London) 221, 179-181

Cooke, R., Franks, K., Ritz-Gold, C. J., Toste, T., Blumenthal, D. K. \& Stull, J. T. (1981) Biophys. J. 33, 235a

Cooke, R., Franks, K. \& Stull, J. T. (1982) FEBS Lett. 144, 33-37

Dhoot, G. K., Perry, S. V. \& Vrbova, G. (1981) Exp. Neurol. 72, 513-530

Gray, S. D. \& Renkin, E. M. (1978) Microvasc. Res. 16, 406-425

Kushmerick, M. J. \& Crow, M. T. (1981) in Basic Biology of Muscle (Twarog, B. M., Levine, R. J. C. \& Dewey, M. M., eds.), pp. 159-172, Raven Press, New York

Manning, D. R. \& Stull, J. T. (1979) Biochem. Biophys. Res. Commun. 90, 164-170

Morgan, M., Perry, S. V. \& Ottaway, J. (1976) Biochem. J. 157, 687-697

Nairn, A. C. \& Perry, S. V. (1979) Biochem. J. 179, 89-97

Pemrick, S. (1980) J. Biol. Chem. 255, 8836-8841

Perry, S. V., Cole, H. A., Frearson, N., Moir, A. J. G., Nairn, A. C. \& Solaro, R. J. (1978) in Proc. FEBS Meet. 11th: Cyclic Nucleotides and Protein Phosphorylation in Cell Regulation (Krause, E. G., Pinna, L. 
\& Wollenberger, A., eds.), pp. 147-159, Pergamon Press, Oxford and New York

Pires, E. M. V. \& Perry, S. V. (1977) Biochem. J. 167, 137-146

Stull, J. T. \& High, C. W. (1977) Biochem. Biophys. Res. Commun. 77, 1078-1083

Stull, J. T., Silver, P. J., Miller, J. R., Blumenthal, D. K., Botterman, B. R. \& Klugg, G. A. (1983) Fed. Proc. Fed. Am. Soc. Exp. Biol. 42, 21-26
Westwood, S. A. \& Perry, S. V. (1981) Biochem. J. 197, 185-195

Westwood, S. A. \& Perry, S. V. (1982) FEBS Lett. 142, 31-34

Westwood, S. A., Hudlicka, O. \& Perry, S. V. (1982) J. Muscle Res. Cell Motil. 3, 511-512

Yagi, K., Yazawa, M., Kakiuchi, S., Oshima, M. \& Uenishi, K. (1978) J. Biol. Chem. 253, 1338-1340 